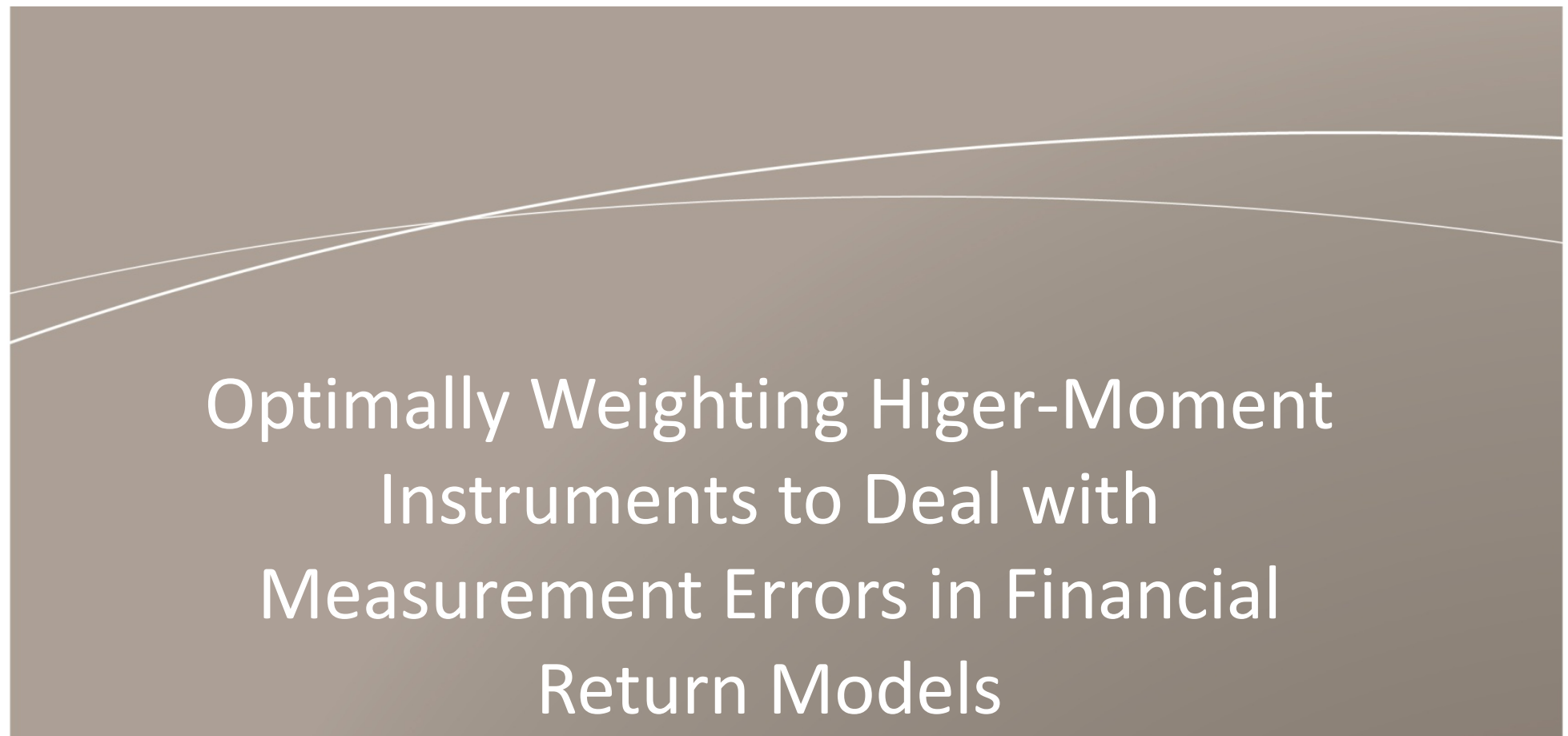

François-Éric Racicot Raymond Théoret

\author{
WORKING PAPER \\ WP.2012.02
}

September 2012

ISSN 0701-3086 


\title{
Optimally weighting higher-moment instruments to deal with measurement errors in financial return models
}

\author{
François-Éric Racicot ${ }^{\dagger}$ \\ Raymond Théoret ${ }^{\dagger \dagger}$
}

Published in Applied Financial Economics, vol. 22, August 2012

September 7, 2012

Accepted at the Southern Finance Association (SFA) annual conference, Charleston (USA),

November 2012.

\begin{abstract}
${ }^{+}$Corresponding author. Telfer School of Management, University of Ottawa, 55 Laurier Street, Ottawa, Ontario, Canada, K1N 6N5; E-mail: Racicot@telfer.uottawa.ca. Tel. (613) 562-5800 ext. 4757. Chaire d'information financière et organisationnelle, ESG-UQAM; Laboratory for Research in Statistics and Probability, LRSP.

\#̈ Professor, University of Quebec (Montreal), (UQAM), 315 Ste-Catherine east, Montreal, Quebec, Canada. H2X 3X2. E-mail: theoret.raymond@uqam.ca. Tel.number: (514) 987-3000 ext. 4417. Professeur associé, Université du Québec (Outaouais); Chaire d'information financière et organisationnelle, ESG-UQAM.
\end{abstract}

Aknowledgements. We would like to thank Ramazan Gençay for his valuable comments. 


\title{
Optimally weighting higher-moment instruments to deal with measurement errors in financial return models
}

\begin{abstract}
Factor loadings are often measured with errors in financial return models. However, these models find applications in many fields of economics and finance. We present a new procedure to optimally weight two well-known cumulant (higher moments) estimators originally designed to deal with errors-in-variables. We develop a new version of the Hausman test which relies on these new instruments in order to build an indicator of measurement errors providing information about the extent of the bias for an estimated coefficient. We apply our new methodology to a well-known financial return model, i.e. the Fama and French (1997) model, over a sample of HFR hedge fund returns, whose distribution is strongly asymmetric and leptokurtic. Our experiments suggest that the market beta is biased by measurement errors, especially at the level of hedge fund strategies. Nevertheless, the alpha puzzle remains robust to our cumulant instruments.
\end{abstract}

Keywords: Cumulant instruments; Asset pricing models; Hausman test; Risk premium; GMM.

JEL classification: C13, C19, C58, G12, G23.

\section{Introduction}

Factor loadings are often measured with errors in financial return models. For instance, many studies have found that the market risk premium is measured with errors whilst estimating the CAPM model (Shanken, 1992; Campbell et al. 1997). In that respect, the most severe critique addressed to the estimation of the market beta concerns the large measurement error associated with the selection of the stock market index used to define the market risk premium (Roll, 1977). Even before the Roll's critique, measurement errors were detected in the two-pass framework implemented by Fama and McBeth (1973) to estimate the CAPM model. The estimation of the APT model is not exempt from measurement errors because the macroeconomic time series used to estimate this model incorporate large errors (Chen et al., 1986). Consumption-based financial returns models like the C-CAPM are also 
plagued with measurement errors associated with the macroeconomic times series used to estimate the models, i.e. consumption and wealth (Cochrane, 2005). Lettau and Ludvigson (2001) have dealt with this problem by building a proxy to the usual factors which enter in the estimation of the C-CAPM. In that respect, they resort to the residuals of the cointegrating vector pulling together consumption, asset wealth and labor income to solve the measurement error problem, these residuals being a strong predictor of market returns. Finally, Meng et al. (2011) propose a simple method they call OLIVE for estimating market betas when factors are measured with errors in the setting of the C-CAPM.

Factor return models find applications in many fields of economics and finance, and especially in corporate finance. For instance they are used in event studies to compute the cumulative abnormal return $(C A R)$ of a merger or more generally to analyze the effect of news (Campbel et al, 1997) on the value of a merger or an acquisition. Moreover, the betas also enter in the computation of the cost of equity, an important ingredient in the valuation of an investment project. Betas also serve to measure systemic risk in many macroeconomic and financial studies, like studies evaluating the effect of financial deregulation on bank systemic risk (Stiroh 2006, Uhde and Michalak 2010). It is thus important to adjust the betas for their measurement errors to avoid the biases associated with these errors.

To deal with measurement errors in financial returns models, we design instruments which are appropriate when the distribution of the variables under study is not Gaussian, i.e. when it is asymmetric or leptokurtic. More precisely, we propose a new weighting of two well-known cumulant instruments originally designed to tackle errors-in-variables, which are the Durbin (1954) and Pal (1980) estimators, and use it as an input to the two-stage least squares (TSLS) and the generalized method of moments (GMM) estimations. Our new optimal instruments are in line with the works of Fuller (1987), Dagenais and Dagenais (1997), Cragg (1997), Lewbel (1997), Coën and Racicot (2007) and Meng et al. (2011). It is 
well-known that the Durbin and Pal's instruments lack robustness (Cheng and Van Ness, 1999) and they thus tend to be neglected by the academics. We show that the robustness of the cumulant instruments can be improved by weighting them optimally. Moreover, we show the equivalence of this procedure with our version of the Hausman artificial regression, which is actually an augmented TSLS, a mapping between two estimation procedures neglected in the literature. To illustrate our method, we estimate the augmented Fama and French (1997) model using a sample of returns defined over 22 hedge fund strategies. The distribution of these returns is highly asymmetric and leptokurtic so the cumulant instruments seem a priori relevant for dealing with the problem of measurement errors in this reduced-form model. In that respect, we particularly focus on the measurement error related to the market risk premium, an unobserved key economic and financial variable, which leads to a correlation between this variable and the model residuals. According to the new indicator for measurement errors we build, we find that the ordinary least-squares (OLS) estimation of the betas may be quite biased, especially at the disaggregated level of the hedge fund strategies.

This article is organized as follows. In section 2, we present the econometric methodology. In section 3, we provide the descriptive statistics of our dataset and the discussion of our empirical results. Section 4 concludes.

\section{Econometric methodology}

\subsection{The Fama and French $(F \& F)$ model}

In this paper, we aim at estimating the well-known augmented F\&F (1997) model, which is:

$$
R_{p t}-R_{f t}=\alpha+\beta_{1}\left(R_{m t}-R_{f t}\right)+\beta_{2} S M B_{t}+\beta_{3} H M L_{t}+\beta_{4} U M D_{t}+\varepsilon_{t}
$$


where $\left(R_{p t}-R_{f t}\right)$ represents the excess return of a portfolio, in our case the portfolio of a hedge fund, over the risk-free rate $\left(R_{f t}\right)$ and $\left(R_{m t}-R_{f t}\right)$ is the market risk premium. $S M B, H M L$ and $U M D$ are long-short mimicking portfolios defined respectively on capitalisation $(S M B)$, bookto-market ratios $(H M L)$ and momentum $(U M D)$. We aim at testing the hypothesis that the factor loadings, $\quad-R_{m t}-R_{f t}, S M B, H M L$ and $U M D$-, are measured with errors. We now present cumulant instruments and an Hausman procedure designed to test our hypothesis.

\subsection{An optimal combination of Durbin and Pal's instruments}

Using the method of moments, Durbin (1954) proposes the following estimator, based on third-order moment and co-moment, to identify the parameter of a simple univariate regression model whose explanatory variable is measured with error:

$$
\hat{\beta}_{d}=\frac{s_{x x y}}{s_{x}^{3}}
$$

where $s_{x x y}$ is a third-order co-moment between the dependent variable $y$ and the explanatory variable $x$ and $s_{x}^{3}$ is the third-order moment of $x$, defined as:

$$
s_{x}^{3}=(n-1)^{-1} \sum_{i=1}^{n}\left(x_{i}-\bar{x}\right)^{3}
$$

and

$$
s_{x x y}=(n-1)^{-1} \sum_{i=1}^{n}\left(y_{i}-\bar{y}\right)\left(x_{i}-\bar{x}\right)^{2}
$$

Subsequently, Pal (1980) derives an estimator based on the fourth-order moment and comoment of the variables of a model, defined as:

$$
\hat{\beta}_{p}=\frac{s_{x x y}-3\left(\sum x^{2} / n\right) s_{x y}}{s_{x}^{4}-3\left(\sum x^{2} / n\right) s_{x}^{2}}
$$


with $s_{x y}=(n-1)^{-1} \sum_{i=1}^{n}\left(y_{i}-\bar{y}\right)\left(x_{i}-\bar{x}\right)$ and $s_{x}^{2}=(n-1)^{-1} \sum_{i=1}^{n}\left(x_{i}-\bar{x}\right)^{2}$. Equation (5) provides an estimator which is a ratio of cumulants, the numerator being a co-cumulant and the denominator, the fourth-order cumulant, a combination of kurtosis and variance (Stuart and Ord, 1994; Malevergne and Sornette, 2005). In the case of a multivariate regression model, the higher moment or cumulant instrumental variables corresponding to these two estimators are respectively (Fuller, 1987; Racicot, 1993):

and

$$
\mathbf{x} \odot \mathbf{x}
$$

$$
\mathbf{x} \odot \mathbf{x} \odot \mathbf{x}-3 \mathbf{x}\left[\mathrm{D} \odot \mathbf{I}_{k}\right]
$$

where $\mathbf{x}$ is the matrix of the explanatory variables expressed in deviation from their mean, $\mathrm{D}=\left(\mathbf{x}^{\mathbf{T}} \mathbf{x} / \mathbf{N}\right)$, a diagonal matrix, and where the symbol $\odot$ stands for the Hadamard product, an element by element matrix multiplication operator. Dagenais and Dagenais (1997) add to these two instruments other cumulants and co-cumulants which were also used previously as instruments by Durbin and Pal in order to identify the parameters of a model containing variables measures with errors. The complete list of the cumulant instrumental variables proposed by Dagenais and Dagenais (1997) is reported in Table 1.

$$
\text { Insert Table } 1 \text { here }
$$

To increase the robustness of these cumulant instruments and reduce their well-known instability, Dagenais and Dagenais rely on Fuller's (1987) instrumental variable (IV) estimator to weight them. They note that the resulting combination seems to perform better than the estimators taken separately. But Dagenais and Dagenais were not very specific on the weighting matrix to use. As shown in Racicot (1993), we can prima facie rely on a Generalized Least-Squares (GLS) weighting of Durbin (1954) and Pal (1980) estimators, respectively equal to $\beta_{D}=\left(\pi_{1}^{T} x\right)^{-1} \pi_{1}^{T} y$ and $\beta_{P}=\left(\pi_{2}^{T} x\right)^{-1} \pi_{2}^{T} y$, for identifying the parameters 
of a model containing measurement errors. In these expressions, all the variables are expressed in deviations from their means with $\pi_{1}=\left[x_{i j}^{2}\right], \pi_{3}=\pi_{2}-3 D\left(x^{T} x / N\right) x^{T}$, and $\pi_{2}=\left[x_{i j}^{3}\right]$. We obtain the new estimator $\beta_{H}$ :

$$
\beta_{H}=\Phi\left[\begin{array}{l}
\beta_{D} \\
\beta_{P}
\end{array}\right]
$$

where $\Phi$ is the GLS weighting matrix. Note that this weighting approach, which relies on GLS as the weighting matrix, is optimal in the Aitken's (1935) sense ${ }^{1}$. However, we rather use the GMM method to weight the Durbin and Pal's estimators, an obviously more efficient method than Dagenais and Dagenais' procedure since we rely on the asymptotic properties of the GMM estimator with respect to the correction of heteroskedasticity and autocorrelation to weight the instruments obtained with GLS.

More specifically, the list of instrumental variables may be extended to other moments and comoments (Table 1) but, in line with Dagenais and Dagenais, we retain the triplet $\left\{z_{0}, z_{1}\right.$, $\left.z_{4}\right\}$, i.e. respectively the constant and the Durbin and Pal's estimators, because the results seem more robust when using this subset of instruments rather than the whole set reported in Table 1. Since the F\&F model we estimate (Equation (1)) comprises four explanatory variables, the number of instruments related to cumulants thus amounts to eight, that is one $z_{1}$ and one $z_{4}$ for each explanatory variable of the F\&F model. In the case of our model, the set of $z$ variables is therefore: $\mathbf{Z}=\left\{z_{0}, z_{11}, z_{12}, z_{13}, z_{14}, z_{41}, z_{42}, z_{43}, z_{44}\right\}$. Our GMM formulation, called GMM-z, obtains ${ }^{2}$ :

$$
\underset{\hat{\beta}}{\arg \min }\left\{n^{-1}\left[\mathbf{Z}^{\mathbf{T}}(\mathbf{y}-\mathbf{X} \hat{\boldsymbol{\beta}})\right]^{T} \mathbf{W} n^{-1}\left[\mathbf{Z}^{\mathbf{T}}(\mathbf{y}-\mathbf{X} \hat{\boldsymbol{\beta}})\right]\right\}
$$

\footnotetext{
${ }^{1}$ Note that we use $\Phi$ as weighting matrix in the GLS estimator (equation (3)). As well-known, this matrix can be replaced by White (1980) asymptotically consistent variance-covariance matrix. The properties of this estimator, named $\beta_{E}$, are discussed in the aforementioned reference. Actually, we implicitly use an augmented version of this estimator in this article.

${ }^{2}$ See Racicot and Théoret (2001), chap.11, for an introduction to the GMM and its applications in finance.
} 
with $\mathbf{W}$, a weighting matrix. This method may provide a robust estimator accounting for the autocorrelation, heteroskedasticity and other usual econometric problems encountered in financial experiments.

\subsection{The Hausman artificial regression}

Using a standard regression model:

$$
\mathbf{Y}=\mathbf{X}^{*} \beta+\boldsymbol{\varepsilon}
$$

assume that $\mathbf{X}^{*}$ is observed with errors. Its observed value, $\mathbf{X}$, is thus equal to:

$$
\mathbf{X}=\mathbf{X}^{*}+\mathbf{v}
$$

with $\mathbf{v}$ being a matrix of random variables assumed to be normally distributed.

Substituting (6) in (5), we have:

$$
\mathbf{Y}=\mathbf{X} \beta+\mathbf{\varepsilon}^{*}
$$

with $\boldsymbol{\varepsilon}^{*}=\boldsymbol{\varepsilon}-\mathbf{v} \boldsymbol{\beta}$

Obviously, $\mathbf{X}$ is correlated with $\boldsymbol{\varepsilon}^{*}$, which creates an endogeneity issue. To tackle this problem, we first regress the explanatory variables $\mathbf{X}$ on the matrix $\mathbf{Z}$, which contains the Durbin and Pal cumulants given by Equations (1) and (2) to obtain $\hat{\mathbf{X}}$ :

$$
\hat{\mathbf{X}}=\mathbf{Z} \hat{\boldsymbol{\theta}}=\mathbf{Z}\left(\mathbf{Z}^{T} \mathbf{Z}\right)^{-1} \mathbf{Z}^{T} \mathbf{X}=\mathbf{P}_{\mathbf{Z}} \mathbf{X}
$$

where $\mathbf{P}_{\mathbf{z}}$ is the conventional "predicted value maker". Having run this regression, we extract the matrix of residuals $\hat{\mathbf{w}}$ :

$$
\hat{\mathbf{w}}=\mathbf{X}-\hat{\mathbf{X}}=\mathbf{X}-\mathbf{P}_{\mathbf{Z}} \mathbf{X}=\left(\mathbf{I}-\mathbf{P}_{\mathbf{Z}}\right) \mathbf{X}
$$

We can write:

$$
\mathbf{X}=\hat{\mathbf{X}}+\hat{\mathbf{w}}
$$


Substituting (10) in (7), we have:

$$
\mathbf{Y}=\hat{\mathbf{X}} \boldsymbol{\beta}+\hat{\mathbf{w}} \boldsymbol{\beta}+\boldsymbol{\varepsilon}^{*}
$$

Since we assume measurement errors so that coefficients estimated by OLS are biased, we replace the coefficients $\boldsymbol{\beta}$ associated with $\hat{\mathbf{w}}$ by the mute coefficient vector $\boldsymbol{\theta}$ :

$$
\mathbf{Y}=\hat{\mathbf{X}} \boldsymbol{\beta}+\hat{\mathbf{w}} \boldsymbol{\theta}+\boldsymbol{\varepsilon}^{*}
$$

To express (12) in terms of $\mathbf{X}$, the vector of observed variables, we replace $\hat{\mathbf{X}}$ by its value given by (10):

$$
\mathbf{Y}=\mathbf{X} \boldsymbol{\beta}+\hat{\mathbf{w}} \lambda+\boldsymbol{\varepsilon}^{*}
$$

where $\boldsymbol{\lambda}=\boldsymbol{\theta}-\boldsymbol{\beta}$ (Pindyck and Rubinfeld, 1998, pp. 195-197). Then we estimate (13) with OLS. An $F$ test on the $\lambda$ coefficients indicates whether they are significant as a group whilst a $t$ test on the individual coefficients indicates whether they are measured with errors. The vector $\boldsymbol{\beta}$ computed by running OLS on (13) is identical to a TSLS estimate, that is:

$$
\hat{\boldsymbol{\beta}}=\hat{\boldsymbol{\beta}}_{\mathrm{TSLS}}=\left(\mathbf{X}^{T} \mathbf{P}_{\mathbf{Z}} \mathbf{X}\right)^{-1} \mathbf{X}^{T} \mathbf{P}_{\mathbf{Z}} \mathbf{Y}
$$

To estimate (13), we use our combination of Durbin and Pal instruments. After substituting the computed $\hat{\mathbf{w}}$ in (13) and running OLS, we obtain a new procedure which is a mapping from the TSLS to the Hausman artificial regression.

To complete this study on measurement errors using cumulants as instruments, a test on the magnitude of these errors is required. To build this test, we rely on the Hausman (Hausman, 1978; McKinnon, 1992; Coën and Racicot, 2007) artificial regression, as given by (13), which we write as:

$$
\mathbf{y}=\mathbf{X} \hat{\boldsymbol{\beta}}_{\mathrm{TSLS}}+\hat{\mathbf{w}} \lambda+\boldsymbol{\varepsilon}^{*}
$$

where $\hat{\mathbf{w}}$ is the vector of the residuals of the regressions of each explanatory variable on the instrument set. As indicated in (15), the vector of estimated coefficients of the explanatory 
variables is identical to the one resulting from a conventional TSLS procedure using the same set of instruments (Spencer and Berk, 1981). This result, overlooked by Dagenais and Dagenais (1997) and other more recent researchers on this topic (Meng et al., 2011), increases the usefulness of Equation (15), and stands for a new way to formulate a TSLS incorporating directly an Hausman errors-in-variables test on each coefficient. Relatedly, in this equation, each explanatory variable $x_{i}$ has its corresponding $w_{i}$. The associated coefficient $\lambda_{i}$ allows detecting measurement errors on $x_{i}$. If it is significantly positive, the corresponding $\beta_{i}$ is overstated in the OLS regression and vice-versa if it is significantly negative. Our estimated coefficients $\lambda_{i}$ are thus new indicators of measurement errors which we formalize by the following empirical relationship:

$$
\operatorname{Spread}_{i s}=\left(\hat{\beta}_{i s, O L S}-\hat{\beta}_{i s, T S L S}\right)=\hat{\pi}_{0}+\hat{\pi}_{1} \hat{\lambda}_{i s}+\xi_{i s} \quad s=1 \text { to } n
$$

where $s$ stands for a specific hedge fund strategy. To sum up, Equation (12) is another way to set up a TSLS but one may prefer this formulation to the one represented by a conventional TSLS in view of the useful information conveyed by this equation.

$$
\text { Insert Table } 2 \text { here }
$$

\subsection{The estimation methods and the instruments}

The full set of the estimation methods we use in this paper is reported in Table 2. As shown in this table, we rely essentially on three estimation methods: the Hausman method we just described, the TSLS and the GMM. To estimate these IV methods, we resort to three groups of instruments: i) the simple higher moment instruments $(\mathrm{hm})$, which are the higher moments of the explanatory variables proposed by Fuller (1987) and Lewbel (1997); ii) the $z$ instruments; iii) and the $d$ instruments, or the distance variables. The $h m$ instruments were originally proposed by Fuller (1987) and Lewbel (1997) in line with Dagenais and Dagenais (1997). This set of instruments is built with the higher-order moments of the dependent and 
explanatory variables up to power 3 . These instruments are computed in deviations from their means. The $d$ instruments, which may be considered as filtered versions of the endogenous variables, are defined as follows:

$$
d_{i t}=x_{i t}-\hat{x}_{i t}
$$

This variable removes some of the nonlinearities embedded in the $x_{i t}$. They are thus a smoothed version of the $x_{i t}$ which might be seen as a proxy for its long-term expected value, the relevant variable in the $\mathrm{F} \& \mathrm{~F}$ model which is theoretically formulated on the explanatory variables expected values. To compute the $\hat{x}_{i t}$ in (17), we perform the following regression:

$$
x_{i t}=\hat{\gamma}_{0}+\mathbf{z} \hat{\boldsymbol{\varphi}}+\varsigma_{t}=\hat{x}_{i t}+\varsigma_{t}
$$

which amounts to run a polynomial adjustment on each explanatory variable.

Summarizing, we resort to three sets of instruments to estimate Equation (1) in this paper: the $h m$, the $z$ (cumulants) and the $d$ (distance) variables. We combine these instruments with our three estimation methods to obtain respectively HAUS-hm, HAU-d, TSLS-hm, TSLS-z, TSLS-d, GMM-hm, GMM-z, and GMM-d³.

\section{Empirical results}

\subsection{Data}

Our sample of hedge funds is composed of the monthly returns of 22 HFR (Hedge Fund Research) indices classified by strategies or groups of strategies. The observation period runs from January 1990 to December 2005, for a total of 192 observations. The risk factors (factor loadings) which appear in the F\&F equation, - that is the market risk

\footnotetext{
${ }^{3}$ Note that we do not report the results of the HAUS-z method because of its low performance.
} 
premium and the three mimicking portfolios: $S M B, H M L$ and $U M D,-$ are drawn from the French's website ${ }^{4}$.

$$
\text { Insert Table } 3 \text { here }
$$

Table 3 provides the descriptive statistics of the 22 HFR selected strategies. The hedge fund indices are sorted by the $R^{2}$ resulting from the OLS estimation of the F\&F model over the period 1990-2005. We note that the F\&F model performs poorly for very specialized hedge fund strategies, like the fixed income arbitrage, convertibles and macro ones. But it seems much more performant to explain the returns of the strategies which dominate the hedge fund industry, which are the equity hedge ${ }^{5}$, fund of funds and equity non hedge strategies.

At an annualized value of $14.5 \%$ over the $1990-2005$ period, the mean return of the hedge fund composite index was higher than the $11.5 \%$ realized by the S\&P500. However, there is a great dispersion of returns over the strategies. The annual return of the short selling index was a meagre $4 \%{ }^{6}$ while the equity hedge index, associated with the most important strategy in the hedge fund industry, displayed a return as high as $17.5 \%$.

A stylised fact about the distribution of hedge fund returns is its degree of kurtosis. Actually, the returns kurtosis of the hedge fund composite index was 5.30 over the 1990-2005 period compared to 3.73 for the S\&P500. In Table 3, kurtosis ranges from a high of 14.71 for the merger arbitrage index to a low of 2.46 for the market timing one. Incidentally, the equity hedge strategy, the most important one in the hedge fund industry, has a kurtosis of 3.92 over the period 1990-2005, a level similar to the S\&P500. Furthermore, the skewness of hedge fund indices is quite high for some strategies like merger arbitrage, event-driven and fixed income arbitrage.

\footnotetext{
${ }^{4}$ The address of the French's website is: http://mba.tuck.dartmouth.edu/pages/faculty/ken.french/data library.html.

5 The equity hedge strategy is often called "long-short strategy".

${ }^{6}$ According to the CAPM, this low return can be explained by the negative beta of this strategy.
} 


\subsection{The empirical models}

The transposition of our general Hausman artificial regression (Equation (13)) to the F\&F model (Equation (1) is:

$$
R_{p t}-R_{f t}=\alpha_{T S L S}+\beta_{1, T S L S}\left(R_{m t}-R_{f t}\right)+\beta_{2, T S L S} S M B_{t}+\beta_{3, T S L S} H M L_{t}+\beta_{4, T S L S} U M D_{t}+\sum_{i=1}^{4} \lambda_{i} \hat{w}_{i t}+\varepsilon_{t}^{*}
$$

The estimated coefficients $\lambda_{i}$, which are equal to $\theta_{i}-\beta_{i}$, allow detecting measurement errors, and their estimated signs indicate whether the corresponding variable is overestimated or underestimated in the OLS regression. Equation (19) is estimated using a hedge fund sample of 22 Hedge Fund Research (HFR) monthly indices (strategies) observed over the period running from January 1990 to December $2005^{7}$. To estimate this equation, the OLS is used as benchmark. The other estimation methods are listed in Table 2.

Table 4 provides the $R^{2}$ of the regressions for the categories of instruments used in this article. We note that $d$ instruments are the most performing in terms of $R^{2}$ followed closely by the $h m$ instruments. The $R^{2}$ of the $z$ instruments are quite lower, so it seems important to transform them in $d$ instruments to increase their performance ${ }^{8}$.

$$
\text { Insert Tables } 4 \text { and } 5 \text { here }
$$

Since the $d$ instruments are new in the literature, we present in Table 5 the regressions of the explanatory variables on the $d$ instruments. These results suggest that they can be

\footnotetext{
${ }^{7}$ We voluntarily choose a sample observed before the US subprime crisis in order to have a more stable observation period, period which corresponds to the Great Moderation in the United States. Nevertheless, the sample includes two crises: the Asian crisis (1997) and the technological bubble (2000-2001). The analysis of the subprime crisis, which was much more important than the two previous ones, is left to further research when more data on the subprime crisis will be available.

${ }^{8}$ In another article, we also estimated the F\&F model with conventional instruments like the predetermined variables of the model and also other usual instruments like the Chen-Roll-Ross (1986) factors, which are the industrial production, the consumer price index, the spread between long and short term bond yields, the spread between BBB and AAA corporate bonds yields and the dividend yield of the S\&P500 (Racicot et al. 2011). The $R^{2}$ was lower than the one obtained with the $\mathrm{z}$ instruments, so we do not rely on the conventional instruments in this article.
} 
considered as strong instruments. In fact, each risk factor has its own instrument to which it is related by an estimated coefficient close to 1 . In that respect, these instruments are uncorrelated to the innovation term of Equation (19). As mentioned before, the $R^{2}$ of these regressions are also quite high.

Insert Table 6 here

Table 6 reports the estimation of Equation (1) for the six selected methods. The results are averaged over the 22 HFR strategies. As justified previously, the coefficients estimated with the TSLS and the Hausman regressions are identical. According to the $R^{2}$, the $z$ instruments are relatively weak compared to the $d$ and $h m$ ones, which display a similar performance. For instance, the $R^{2}$ obtained with the TSLS-z and GMM-z are both equal to 0.32 while this statistic is about 0.45 using the two other categories of instruments. Moreover, the estimation methods which seem to perform the best are the HAUS-hm and HAUS-d methods, their $R^{2}$ being respectively 0.48 and 0.47 . Furthermore, the DW statistic does not signal any serious residuals autocorrelation problem regardless the estimation method used.

\subsubsection{OLS estimations}

Let us look more closely at the OLS results more closely. First, the alpha of Jensen ${ }^{9}$, a measure of the absolute return, is high over the estimation period, being equal to $0.43 \%$ on a monthly basis and significant at the $1 \%$ threshold. This is in line with the studies on the alpha puzzle prevailing in the hedge fund industry. Second, not surprisingly, the estimated market beta is low in the hedge fund industry, being estimated at 0.20 during the period 1990-2005 and significant at the $1 \%$ level. The sensitivity of the returns of the representative hedge fund to the stock market is thus low. The estimated coefficient of $S M B$ is equal to 0.13 and significant at the $1 \%$ threshold. Then, there seems to be a herd-like behaviour in the hedge

\footnotetext{
${ }^{9}$ The alpha is the constant in the F\&F model.
} 
fund industry regarding the investment in small firms (Haiss, 2005) ${ }^{10}$. This can be explained by the small firm anomaly, i.e. a higher risk-adjusted expected return for small capitalization stocks versus larger capitalization stocks. Indeed, small firms are confronted to a severe asymmetry problem at the level of financial reporting, which explains in part the small firm anomaly ${ }^{11}$. The sensitivity of hedge funds to $H M L$ is smaller than to $S M B$, the coefficient being equal to 0.07 and significant at the $1 \%$ threshold. Finally, consistent with other studies, the sensitivity of hedge funds to the momentum of stock market, $U M D$, is much lower, at 0.04 and significant at the $1 \%$ threshold. Therefore, the representative hedge fund does not seem to track the stock market trend.

\subsubsection{The IV estimations}

Using the whole sample of hedge funds strategies, a comparison of the average estimated coefficients of the OLS to those of the IV methods first suggests that the alpha is not very sensitive to the estimation method used. For instance, it is equal to 0.4283 when using the OLS method, with a low 0.3713 associated with the TSLS-z method and a high of 0.4309 associated with the GMM-hm method. Moreover, these coefficients are all significant at the $1 \%$ level, regardless of the estimation method used. The alpha puzzle is thus robust to the IV estimation methods we consider. Second, our estimations allow detecting nonnegligible measurement errors. In this respect, the market risk premium displays the highest measurement errors. For instance, computed over the 22 HFR indices, the mean beta is equal to 0.23 and significant at the $1 \%$ level. The range of the estimated betas varies from a low of 0.19, obtained with the GMM-d and the TSLS-d methods, to a high of 0.29 , associated with the TSLS-z. There is thus a wide range of possible values for the market beta according to the IV method used. The importance of the bias caused by measurement error may be captured by

\footnotetext{
${ }^{10}$ This result is also consistent hedge investment in techno stocks and startups companies during the dotcom boom (McGuire et al., 2005).

${ }^{11}$ Note that small firms are usually riskier than big ones but the small firm anomaly refers to the risk-adjusted return.
} 
the coefficient of the respective $w$ of the market risk premium. Using the HAUS-d method, this coefficient is equal to 0.06 but insignificant, which, according to this test, suggests an overstatement of the hedge fund beta by the OLS method. Note that there are seven strategies for which the coefficient associated with $w$ is significant at the $5 \%$ threshold, with a maximum $t$-statistic of 3.58 for one strategy, which suggests a serious overstatement for the associated strategy in this case (Table 6). Using the HAUS-hm, the coefficient of the $w$ artificial variable associated with the market risk premium is equal to -0.05 and significant at the level of $10 \%$, which suggests that OLS understates the beta. And actually, the corresponding GMM-hm method attributes a higher beta to the representative hedge fund than the GMM-d one, 0.21 versus 0.19 . In that instance, with the HAUS-hm method, there are nine strategies displaying a significant coefficient for the $w$ variable at the usual thresholds with a maximum $t$ value of 5.19, which suggests a serious understatement for the associated strategy. Furthermore, for the HAUS-hm strategy, there are also nine strategies having an $U M D$ coefficient significantly measured with errors.

However note that the direction of the bias related to the coefficient of the market risk premium is ambiguous. Indeed, the $h m$ instruments give rise to a systematic overestimation of the market beta whilst the $d$ instruments entail a systematic underestimation of the beta. The results are thus sensitive to the instruments used to deal with the measurement errors problem. They cast doubt on previous studies dealing with the endogeneity issue embedded in the F\&F model with only one kind of instruments. It seems that some a priori information, like Bayesian or inside information, is required to help identify the direction of the bias caused by measurement errors, which is the case with many economic reduced-form models.

Summarizing, the two Hausman methods reported in Table 6 provide consistent results and detect seemingly more measurements errors at a disaggregated level, as expected, aggregation having a tendency to reduce measurements errors among strategies. Indeed, it is 
well-known in finance that aggregation of securities diversifies (dilutes) idiosyncratic risk. The same phenomenon is at play here.

$$
\text { Insert Table } 7 \text { here }
$$

\subsubsection{The measurement errors indicator}

To test for the relevance of our estimation errors indicator for the market beta of our hedge fund strategies, we rely on the estimation of Equation (16). Table 7, which is built with higher moment instruments, shows the high positive correlation between the coefficients of measurement errors, the $\lambda$, and the spread between the coefficients estimated with the OLS and HAUS-hm methods. Regressing the spread on the $\lambda$, we obtain the following result:

$$
\text { spread_hm }=\left(\hat{\beta}_{R_{m}-R_{f}, O L S}-\hat{\beta}_{R_{m}-R_{f}, T S L S}\right)_{\mathrm{s}}=\underset{(-0.11)}{0.0003}+\underset{(19.73)}{0.562} \hat{\lambda}_{s}+\xi_{s}
$$

with a $R^{2}$ of 0.95 and the $t$-statistics of the estimated coefficients reported in parentheses. This equation reveals that there are significant measurement errors for the conventional beta at the disaggregated level for the hedge fund strategies. Figure 1, which plots Equation (20) along

with the observed values, shows the close relationship between the spread and $\lambda$ when using higher moments instruments.

\section{Insert Figure 1 about here}

Similarly, Figure 2 plots the results of the estimation of the same equation with the optimal $d$ instruments. Equation (21) obtains:

$$
\text { spread_d }=\left(\hat{\beta}_{R_{m}-R_{f}, O L S}-\hat{\beta}_{R_{m}-R_{f}, T S L S}\right)_{\mathrm{s}}=\underset{(1.97)}{0.001}+\underset{(39.60)}{0.165} \hat{\lambda}_{s}+\xi_{s}
$$

with a $R^{2}$ even higher, equal to 0.99 . The figure shows that the observation dots are better aligned along the regression line with the $d$ instruments than with the $h m$ ones, which translates in less volatility of the observations around the fitted line. This suggests that the $d$ 
instruments may be more robust than the $h m$ instruments, the former being computed with an optimal weighting procedure.

\section{Insert Figure 2 here}

\section{Conclusion}

In this paper, we found that cumulant and higher moment instruments may be considered as robust instruments to estimate models of financial or economic variables whose distributions are, like hedge fund returns, asymmetric or leptokurtic. In that respect, we analyze three sets of instruments: the higher moments instruments $(\mathrm{hm})$, the cumulants instruments $(z)$ and a new set of instruments, the distance $(d)$ instruments. The $\mathrm{z}$ instruments, which are a combination of Durbin and Pal's cumulants instruments, provide quite erratic results. The $h m$ instruments perform much better but the $d$ instruments display the best results. These instruments are based on the distance between the observed endogenous variables and their fitted values computed with our cumulants instruments. They present the advantage of filtering the endogenous variables, removing their nonlinearities, which lead to more stable instruments. Our tests show that these instruments have a correlation close to 1 with their corresponding explanatory variable and are orthogonal to the other variables of our models.

We combined our three sets of instruments, - the $z, h m$ and $d$ instruments -, with two IV estimation methods, the TSLS and the GMM. We also developed a new form of TSLS which directly embeds a measurement error test. This TSLS is based on the Hausman artificial regression. It allows us to define a new indicator of measurement errors.

Our experiments show that there may be important measurement errors in the estimation of the factor loadings of the F\&F model when running this model with hedge fund returns. Measurement errors are especially important for the estimation of the market beta, particularly at the strategy level. Nevertheless, the direction of the error is sensitive to the 
kind of instruments used. In other words, the methods detect quite well the extent of the measurement errors but are quite weak to detect whether there is an overstatement or an understatement of the model coefficients estimated with the OLS. This result casts doubt on previous studies dealing with the endogeneity issue embedded in the F\&F model with only one kind of instruments. It seems that some a priori information, like Bayesian or inside information, is required to help identify the direction of the bias due to measurement errors, a problem shared by many economic reduced-form models. Furthermore, the new indicator of measurement errors we use, which is derived from the Hausman artificial regression, proved to be quite efficient to detect such errors, especially in the case of the market risk premium, a variable known to be measured with error. Care must thus be taken when using OLS betas as measures of systemic risk.

\section{References}

Aitken, A.C., 1935. On least squares and linear combination of observations. Proceedings of the Royal Society of Edimburgh 55, 42-48.

Campbell, J.Y., Lo, A.W., MacKinlay, A.C., 1997. The econometrics of financial markets. Princeton University Press, Princeton.

Chen, N.F., Roll, R., Ross, S., 1986. Economic forces and the stock market. Journal of Business 59, 572-621.

Cheng, C.L., Van Ness, J.W., 1999. Regression with Measurement Error, Arnold.

Cochrane, J.H., 2005. Asset Pricing. $2^{\text {nd }}$ Edition. Princeton University Press, Princeton.

Coën, A., Racicot, F.E., 2007. Capital asset pricing models revisited: Evidence from errors in variables. Economics Letters 95, 443-450.

Cragg, J.G., 1997. Using higher moments instruments to estimate the simple errors-invariables model. Rand Journal of Economics 28, S71-S91.

Dagenais, M.G., Dagenais, D.L., 1997. Higher moment estimators for linear regression models with errors in the variables. Journal of Econometrics 76, 193-221.

Durbin, J., 1954. Errors in variables. International Statistical Review 22, 23-32.

Fama, E.F., French, K.R., 1997. Industry costs of equity. Journal of Financial Economics 43, 153-193.

Fama, E., McBeth, J., 1973. Risk, returns and equilibrium: Empirical tests. Journal of Political Economy 81, 607-636.

Fuller, W.A., 1987. Measurement Error Models, John Wiley \& Sons.

Geary, R.C., 1942. Inherent relations between random variables. Proceedings of the Royal Irish Academy 47, 23-76. 
Haiss, P. R., 2005. Banks, herding and regulation: A review and synthesis. Working paper, Europe Institute.

Hausman, J.A., 1978. Specification tests in econometrics. Econometrica 46, 1251-1271.

Lettau, M., Ludvigson, S., 2001. Resurrecting the (C)CAPM: A cross-sectional test when risk premia ar time-varying. Journal of Political Economy 109, 1238-1287.

Lewbel A., 1997. Constructing instruments for regressions with measurement error when no additional data are available, with an application to patents and R\&D. Econometrica 65, 1201-1213.

MacKinnon, J.G., 1992. Model specification tests and artificial regressions. Journal of Economic Literature 30, 102-146.

Malevergne, Y., Sornette, D., 2005. Higher-moment portfolio theory: Capitalizing on behavioural anomalies of stock markets. Journal of Portfolio Management 31, 49-55.

McGuire, P. , Remolona, E., Tsatsaronis, K., 2005. Time-varying exposures and leverage in hedge

funds. BIS Quarterly Review 1, 59-72.

Meng, J.G., Hu, G., Bai, J., 2011. Olive: A simple method for estimating betas when factors are measured with errors. Journal of Financial Research 34(1), 27-60.

Pal., M., 1980. Consistent moment estimators of regression coefficients in the presence of errors in variables. Journal of Econometrics 14, 349-364.

Pindyck, R.S., Rubinfeld, D.L., 1998. Econometric Models and Economic Forecasts. $4^{\text {th }}$ edition. IrwinMcGraw-Hill.

Racicot F.E., 1993. Techniques alternatives d'estimation et tests en présence d'erreurs de mesure sur les variables explicatives. M.Sc. thesis. University of Montreal: Department of economics. https://papyrus.bib.umontreal.ca/jspui/bitstream/1866/1076/1/a1.1g638.pdf.

Racicot, F.E., Théoret, R., 2001. Traité d'économétrie financière. Presses de 1'Université du Québec, Ste-Foy.

Racicot, F.E., Théoret, R., Coën, A., 2011. A new empirical version of the Fama and French model based on the Hausman specification test: An application to hedge funds. Journal of Derivatives and Hedge Funds 16, 278-302.

Roll, R., 1977. A critique of asset pricing theory's test: Part 1. Journal of Financial Economics 4, 129-176.

Shanken, J., 1992. On the estimation of beta-pricing models. Review of Financial Studies 5, $1-34$.

Spencer, D.E., Berk, K.N., 1981. A limited information specification test. Econometrica 49, 1079-1085.

Stiroh, K.J., 2006. A portfolio view of banking with interest and noninterest activities. Journal of Money, Credit and Banking 38, 1351-1361.

Stuart, A., Ord, K., 1994. Kendall's Advanced Theory of Statistics, vol. 1, Arnold.

Uhde, A., Michalak, T.C. 2010. Securitization and systematic risk in European banking: Empirical evidence. Journal of Banking \& Finance 34, 3061-3077.

White, H., 1980. A heteroscedasticity-consistent covariance matrix estimator and a direct test for heteroscedasticity. Econometrica 48, 817-838. 
Table 1 List of the cumulant instrumental variables proposed by Dagenais and Dagenais $(1997)^{\dagger}$

\begin{tabular}{|c|c|}
\hline $\mathbf{z}_{0}$ & $\mathbf{l}_{\mathrm{N}}$ \\
\hline $\mathbf{z}_{1}$ & $\mathbf{x} \odot \mathbf{x}$ (Durbin) \\
\hline $\mathbf{z}_{2}$ & $\mathbf{x} \odot \mathbf{y}$ \\
\hline $\mathbf{z}_{3}$ & $\mathbf{y} \odot \mathbf{y}$ \\
\hline $\mathbf{z}_{4}$ & $\mathbf{x} \odot \mathbf{x} \odot \mathbf{x}-3 \mathbf{x}\left[\mathbf{E}\left(\mathbf{x}^{\prime} \mathbf{x} / \mathbf{N}\right) \odot \mathbf{I}_{k}\right] \quad(P a l)$ \\
\hline $\mathbf{z}_{5}$ & $\mathbf{x} \odot \mathbf{x} \odot \mathbf{y}-\mathbf{2 x}\left[\mathbf{E}\left(\mathbf{x}^{\prime} \mathbf{y} / \mathbf{N}\right) \odot \mathbf{I}_{\mathbf{k}}\right]-\mathbf{y}\left\{\mathbf{l}_{\mathrm{k}}{ }_{\mathrm{k}}\left[\mathbf{E}\left(\mathbf{x}^{\prime} \mathbf{x} / \mathbf{N}\right) \odot \mathbf{I}_{\mathbf{k}}\right]\right\}$ \\
\hline $\mathbf{z}_{6}$ & $x \odot y \odot y-x\left[E\left(y^{\prime} y / N\right]-2 y\left[E\left(y^{\prime} x / N\right]\right.\right.$ \\
\hline $\mathbf{z}_{7}$ & 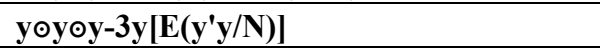 \\
\hline
\end{tabular}

$\dagger \mathbf{t}$ stands for a vector of one. $\mathbf{I}_{\mathbf{k}}$ is the identity matrix of dimension $(\mathrm{k} \mathrm{x} \mathrm{k})$. ๑stands for the Hadamard product. 
Table 2 List of higher moment and cumulant estimators used to correct measurement errors ${ }^{\dagger}$

\begin{tabular}{|c|c|}
\hline Method & Instruments \\
\hline HAUS-hm & higher moments: $\left\{x_{t-1}, x_{t}^{2}, x_{t}^{3}, y_{t}^{2}\right\}$ \\
\hline HAUS-d & the distance variables: $\left\{\mathrm{d}_{1}, \ldots, \mathrm{d}_{4}\right\}$ \\
\hline TSLS-hm & higher moments: $\left\{x_{t-1}, x_{t}^{2}, x_{t}^{3}, y_{t}^{2}\right\}$ \\
\hline TSLS-z & $\begin{array}{c}\left\{\mathrm{Z}_{0}, \mathrm{z}_{11}, \ldots \mathrm{Z}_{14}, \mathrm{Z}_{41}, \ldots, \mathrm{Z}_{44}\right\}, \text { which are the Durbin and Pal } \\
\text { instruments (cumulants) }\end{array}$ \\
\hline TSLS-d & the distance variables: $\left\{d_{1}, \ldots, d_{4}\right\}$ \\
\hline GMM-hm & higher moments: $\left\{x_{t-1}, x_{t}^{2}, x_{t}^{3}, y_{t}^{2}\right\}$ \\
\hline GMM-z & $\begin{array}{c}\left\{\mathrm{Z}_{0}, \mathrm{z}_{11}, \ldots \mathrm{Z}_{14}, \mathrm{Z}_{41}, \ldots, \mathrm{Z}_{44}\right\}, \text { which are the Durbin and Pal } \\
\text { instruments (cumulants) }\end{array}$ \\
\hline GMM-d & the distance variables: $\left\{d_{1}, \ldots d_{4}\right\}$ \\
\hline
\end{tabular}

${ }^{\dagger}$ The variables which enter in the computation of higher moments and cumulants are expressed in deviation from their mean. The Haus-hm and Haus-d methods are the Hausman artificial regressions using respectively as instruments the higher moments and cumulants of the explanatory variables. Three two-stage least squares methods are used, differing by their instruments: higher moments $(\mathrm{hm}), z$ instruments $(z)$ (Table 1), and distance instruments $(d)$. We use the same methods with the GMM. Note that for the GMM estimations, the weighting matrix used to weight the moment conditions is the Newey West one. 
Table 3 Descriptive statistics of the HFR indices, 1990-2005*

\begin{tabular}{|c|c|c|c|c|c|c|}
\hline & $\begin{array}{c}\text { Mean return } \\
\text { (ann) }\end{array}$ & Median (ann) & s.d. (ann) & skewness & kurtosis & $\mathbf{R}^{2}$ \\
\hline Fixed income arbitrage & 8.14 & 8.52 & 5.22 & -1.07 & 13.93 & 0.01 \\
\hline Funds of funds (FoF) Market defensive & 9.43 & 8.82 & 5.89 & 0.18 & 4.35 & 0.06 \\
\hline Convertibles & 10.00 & 11.64 & 3.74 & -1.27 & 6.61 & 0.16 \\
\hline Macro & 13.51 & 11.28 & 8.77 & 0.43 & 3.82 & 0.19 \\
\hline Relative Value Arbitrage & 11.70 & 10.68 & 3.70 & 0.08 & 10.30 & 0.28 \\
\hline FoF Conservative & 8.31 & 8.94 & 3.23 & -0.47 & 6.50 & 0.31 \\
\hline Merger Arbitrage & 10.48 & 12.9 & 4.59 & -2.61 & 14.71 & 0.32 \\
\hline Fixed income high yield & 8.65 & 8.82 & 6.16 & -0.44 & 8.98 & 0.34 \\
\hline Market Neutral Statistical Arbitrage & 8.00 & 8.94 & 3.95 & -0.26 & 3.67 & 0.36 \\
\hline Fixed income total & 11.70 & 11.76 & 4.14 & 0.06 & 6.35 & 0.40 \\
\hline Equity Market Neutral & 9.76 & 9.12 & 3.60 & -0.10 & 5.16 & 0.42 \\
\hline FoF diversified & 9.02 & 8.46 & 5.94 & -0.10 & 7.30 & 0.42 \\
\hline Distressed Securities & 14.42 & 13.56 & 6.14 & -0.67 & 8.46 & 0.44 \\
\hline FoF total & 10.25 & 9.72 & 4.60 & -0.33 & 7.13 & 0.45 \\
\hline FoF strategic & 12.85 & 14.82 & 8.91 & -0.38 & 6.74 & 0.49 \\
\hline Market timing & 12.94 & 11.94 & 7.25 & 0.14 & 2.46 & 0.58 \\
\hline Event Driven & 14.72 & 16.98 & 7.07 & -1.24 & 7.60 & 0.73 \\
\hline Sector & 19.38 & 21.66 & 12.78 & 0.11 & 6.19 & 0.75 \\
\hline Equity hedge & 17.46 & 19.38 & 9.90 & 0.17 & 3.92 & 0.76 \\
\hline Short selling & 4.00 & -0.90 & 23.10 & 0.26 & 5.10 & 0.78 \\
\hline Equity non hedge & 16.91 & 21.84 & 15.45 & -0.42 & 3.98 & 0.91 \\
\hline Average & 11.51 & 11.85 & 7.34 & -0.38 & 6.82 & 0.45 \\
\hline Hedge fund weighted composite & 14.51 & 18.18 & 7.01 & -0.51 & 5.30 & 0.82 \\
\hline S\&P500 & 11.09 & 15.06 & 14.31 & -0.45 & 3.73 & \\
\hline
\end{tabular}

* The statistics reported in this table are computed using the monthly returns of the HFR indices over the period running from January 1990 to December 2005 . The weighted composite index is computed over the whole set of the HFR indices. The $R^{2}$ are obtained with the OLS estimations of the F\&F model for each strategy. The strategies are sorted by increasing value of $R^{2}$. 
Table 4 Adjusted $R^{2}$ of the OLS regressions of the explanatory variables of the F\&F model on instrument catogories ${ }^{*}$

\begin{tabular}{l|c|c|c|c|c}
\hline & classical & hm with $\boldsymbol{y}^{2}$ & hm without $\boldsymbol{y}^{2}$ & $\boldsymbol{z}$ & $\boldsymbol{d}$ \\
\hline $\boldsymbol{r}_{\boldsymbol{m}}$ & 0.10 & 0.61 & 0.56 & 0.21 & 0.80 \\
$\boldsymbol{S M B}$ & 0.11 & 0.48 & 0.55 & 0.35 & 0.62 \\
$\boldsymbol{H} \boldsymbol{M} \boldsymbol{L}$ & 0.09 & 0.61 & 0.64 & 0.23 & 0.74 \\
$\boldsymbol{U M D}$ & 0.05 & 0.53 & 0.51 & 0.32 & 0.65 \\
\hline
\end{tabular}

* In this table, $h m$ stands for higher moments. 
Table 5 Regression of the explanatory variables of the F\&F model on the dinstruments ${ }^{\dagger}$

\begin{tabular}{|c|c|c|c|c|}
\hline & $\boldsymbol{R}_{m}-\boldsymbol{R}_{f}$ & $S M B$ & $H M L$ & UMD \\
\hline \multirow[t]{2}{*}{$d_{1}$} & 1.00 & 0.01 & -0.01 & -0.01 \\
\hline & 23.93 & 0.27 & -0.15 & -0.12 \\
\hline \multirow[t]{2}{*}{$d_{2}$} & 0.01 & 1.00 & -0.01 & -0.01 \\
\hline & 0.20 & 17.01 & -0.05 & -0.04 \\
\hline \multirow[t]{2}{*}{$d_{3}$} & 0.01 & 0.00 & 0.99 & -0.01 \\
\hline & 0.17 & 0.03 & 19.30 & -0.03 \\
\hline \multirow[t]{2}{*}{$d_{4}$} & -0.01 & -0.01 & 0.01 & 1.00 \\
\hline & -0.08 & -0.02 & 0.02 & 18.49 \\
\hline Adjusted $R^{2}$ & 0.80 & 0.62 & 0.74 & 0.65 \\
\hline$D W$ & 1.87 & 2.52 & 2.40 & 2.22 \\
\hline
\end{tabular}

$\dagger$ Each explanatory variable is regressed on the four $d$ instrumental variables built according to Equation (22). The regression coefficient of an explanatory variable on its corresponding $d$ is bolded. The coefficient $t$ statistics are in italics. 
Table 6 F\&F model estimated with OLS and several cumulant IV methods over 22 HFR indices, $1990-2005^{\dagger}$

\begin{tabular}{|c|c|c|c|c|c|c|c|c|c|c|c|}
\hline & $c$ & $r m$ & $S M B$ & $H M L$ & $U M D$ & $\widehat{w}_{r m_{-}}$ & $\widehat{w}_{S M B}$ & $\widehat{w}_{H M L}$ & $\widehat{w}_{U M D}$ & $R^{2}$ & $D W$ \\
\hline \multirow[t]{2}{*}{ OLS } & 0.4283 & 0.2016 & 0.1226 & 0.0739 & 0.0378 & & & & & 0.45 & 1.60 \\
\hline & 4.22 & 10.42 & 5.51 & 2.55 & 2.70 & & & & & & \\
\hline \multirow[t]{2}{*}{ TSLS-hm } & 0.4030 & 0.2304 & 0.1727 & 0.1346 & 0.0118 & & & & & 0.45 & 1.61 \\
\hline & 3.44 & 8.15 & 4.26 & 2.23 & 2.22 & & & & & & \\
\hline \multirow[t]{2}{*}{ TSLS-z } & 0.3713 & 0.2884 & 0.1186 & 0.0912 & 0.0375 & & & & & 0.32 & 1.70 \\
\hline & 2.71 & 4.70 & 1.75 & 1.30 & 1.47 & & & & & & \\
\hline \multirow[t]{2}{*}{ TSLS-d } & 0.4241 & 0.1910 & 0.1175 & 0.0734 & 0.0506 & & & & & 0.45 & 1.61 \\
\hline & 3.44 & 6.98 & 3.55 & 1.90 & 2.01 & & & & & & \\
\hline GMM-hm & 4.20 & 7.50 & 6.60 & 2.80 & 2.93 & & & & & & \\
\hline \multirow[t]{2}{*}{ GMM-z } & 0.3963 & 0.2799 & 0.1106 & 0.0867 & 0.0380 & & & & & 0.32 & 1.70 \\
\hline & 2.53 & 4.20 & 2.05 & 1.51 & 1.36 & & & & & & \\
\hline \multirow[t]{2}{*}{ GMM-d } & 0.4241 & 0.1910 & 0.1175 & 0.0734 & 0.0506 & & & & & 0.45 & 1.61 \\
\hline & 3.44 & 8.15 & 4.26 & 2.23 & 2.22 & & & & & & \\
\hline \multirow[t]{2}{*}{ Mean } & 0.4090 & 0.2341 & 0.1331 & 0.0928 & 0.0317 & & & & & 0.40 & 1.64 \\
\hline & 3.42 & 7.19 & 4.07 & 2.10 & 2.15 & & & & & & \\
\hline HAUS-hm & 0.4030 & 0.2304 & 0.1727 & 0.1346 & 0.0118 & -0.0482 & -0.0803 & -0.0736 & 0.0578 & 0.48 & 1.62 \\
\hline$t \max$ & & & & & & 5.19 & 4.94 & 4.85 & 2.73 & & \\
\hline$t \mathrm{sd}$ & & & & & & 1.30 & 1.48 & 1.29 & 0.79 & & \\
\hline \# signif. strat & & & & & & 9 & 6 & 6 & 9 & & \\
\hline \multirow[t]{2}{*}{ HAUS-d } & 0.4241 & 0.1910 & 0.1175 & 0.0734 & 0.0506 & 0.0600 & 0.0093 & 0.0149 & -0.0308 & 0.47 & 1.61 \\
\hline & 4.43 & 9.54 & 4.90 & 2.30 & 2.53 & 1.49 & 0.69 & 1.04 & 1.03 & & \\
\hline$t \min$ & & & & & & 0.01 & 0.07 & 0.02 & 0.17 & & \\
\hline$t \max$ & & & & & & 3.58 & 1.66 & 2.87 & 2.28 & & \\
\hline$t \mathrm{sd}$ & & & & & & 1.11 & 0.48 & 0.77 & 0.56 & & \\
\hline \# signif. strat & & & & & & 7 & 0 & 4 & 2 & & \\
\hline
\end{tabular}

${ }^{\dagger}$ The estimated equation is given by Equation (1). The methods we use are explained in Table 2. Coefficient $t$-statistics are in italics. For the Haus-hm and the Haus-d estimations, we provide the minimum and maximum $t$-statistics over all the strategies analyzed, and the number of strategies for which the $t$-statistics is significant at the $95 \%$ confidence level. The J tests, not reported here to simplify the table, indicate that the instruments sets used to run the GMM are well identified. 
Table 7 Spread and HAUS-hm test for the coefficient of the market risk premium ${ }^{\dagger}$

\begin{tabular}{|c|c|c|c|c|c|}
\hline & OLS & HAUS-hm & Spread & $\lambda$ & $t(\lambda)$ \\
\hline Distressed & 0.24 & 0.37 & -0.13 & -0.19 & -3.64 \\
\hline Fixed income high yield & 0.24 & 0.34 & -0.10 & -0.14 & -2.33 \\
\hline Merger Arbitrage & 0.19 & 0.28 & -0.09 & -0.18 & -3.98 \\
\hline Event Driven & 0.40 & 0.49 & -0.09 & -0.14 & -3.29 \\
\hline FoF strategic & 0.34 & 0.41 & -0.07 & -0.14 & -1.77 \\
\hline Convertibles & 0.10 & 0.17 & -0.07 & -0.11 & -2.80 \\
\hline Fixed income arb. & -0.03 & 0.04 & -0.06 & -0.10 & -1.56 \\
\hline FoF diversified & 0.22 & 0.26 & -0.05 & -0.10 & -1.77 \\
\hline FWC & 0.38 & 0.42 & -0.04 & -0.07 & -1.86 \\
\hline FOF Conservative & 0.12 & 0.16 & -0.04 & -0.08 & -2.47 \\
\hline Relative Value Arb. & 0.12 & 0.16 & -0.04 & -0.07 & -1.87 \\
\hline FoF Market def. & 0.04 & 0.07 & -0.03 & -0.11 & -1.56 \\
\hline Fund of Funds & 0.18 & 0.21 & -0.03 & -0.07 & -1.73 \\
\hline Equity Market Neutral & 0.09 & 0.11 & -0.03 & -0.05 & -1.61 \\
\hline Fixed income tot. & 0.16 & 0.19 & -0.03 & -0.03 & -0.67 \\
\hline Equity non hedge & 0.85 & 0.87 & -0.02 & -0.02 & -0.29 \\
\hline MN Stat Arbitrage & 0.19 & 0.19 & 0.00 & 0.01 & 0.38 \\
\hline Equity hedge & 0.49 & 0.48 & 0.01 & 0.01 & 0.14 \\
\hline Macro & 0.28 & 0.26 & 0.03 & 0.06 & 0.65 \\
\hline Sector & 0.51 & 0.46 & 0.05 & 0.06 & 0.81 \\
\hline Short selling & -1.02 & -1.08 & 0.07 & 0.09 & 0.71 \\
\hline Market timing & 0.35 & 0.21 & 0.14 & 0.28 & 5.19 \\
\hline
\end{tabular}

The spread (measurement error) is the difference between the OLS coefficient and the corresponding Hausman coefficient resulting from the estimation of the Hausman artificial regression (Equation 21). For each spread, we provide the coefficient $\lambda$ of the corresponding artificial variable. The funds having a significant $\lambda$ at the $10 \%$ level are bold-faced. Note the strong positive relationship between the spread and $\lambda$, the strategies being reported in increasing order of the spread. 
Figure 1 Relation between the spread and the corresponding $\lambda$ estimated with HAUS-hm for the market risk premium

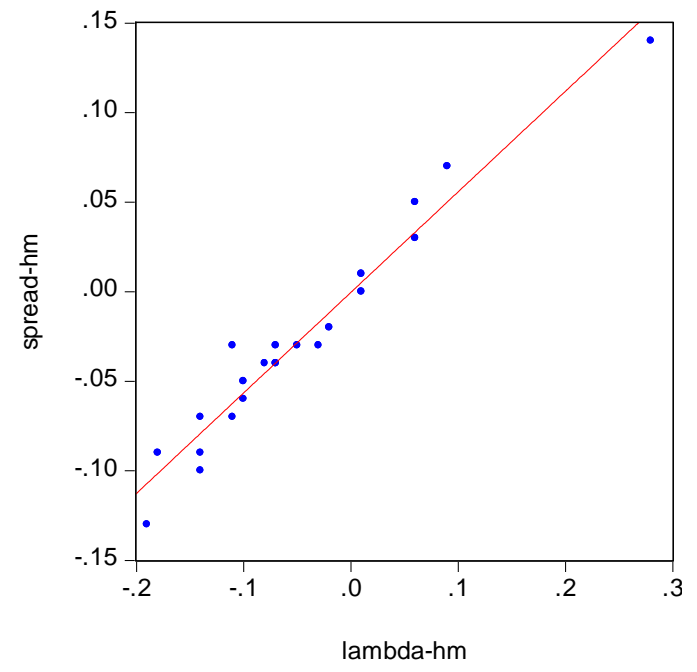

Figure 2 Relation between the spread and the corresponding $\lambda$ estimated with HAUS-d for the market risk premium

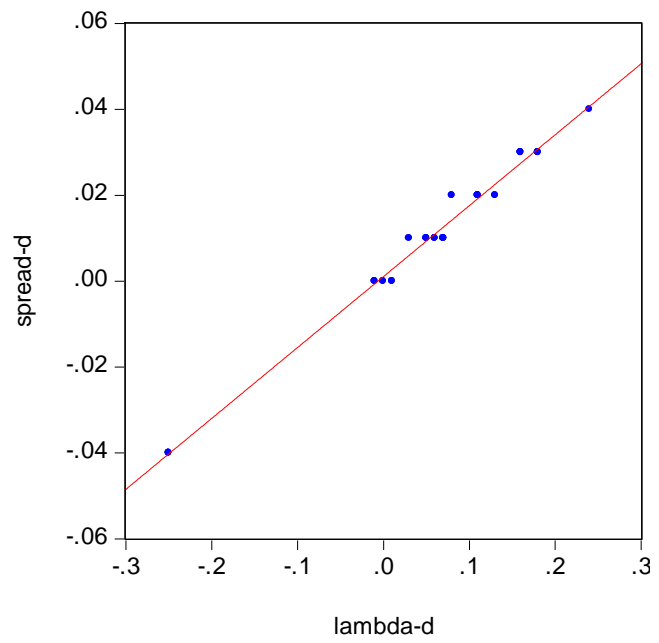

